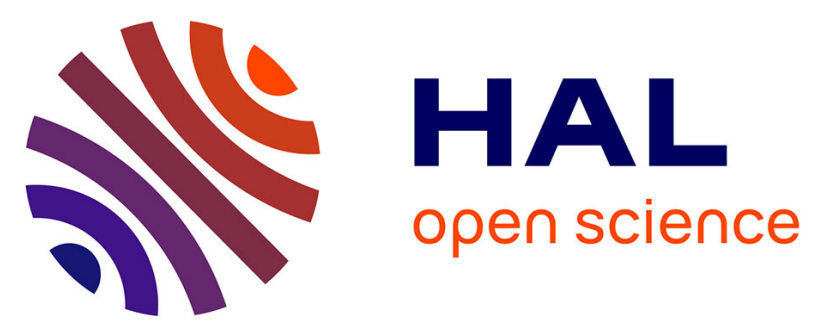

\title{
Resource Allocation in Downlink Non-orthogonal Multiple Access (NOMA) for Future Radio Access
}

Marie Rita Hojeij, Joumana Farah, Charbel Abdel Nour, Catherine Douillard

\section{To cite this version:}

Marie Rita Hojeij, Joumana Farah, Charbel Abdel Nour, Catherine Douillard. Resource Allocation in Downlink Non-orthogonal Multiple Access (NOMA) for Future Radio Access. VTC 2015 spring: IEEE 81st Vehicular Technology Conference, May 2015, Glasgow, United Kingdom. pp.1 - 6, 10.1109/VTCSpring.2015.7146056 . hal-01184177

\section{HAL Id: hal-01184177 https://hal.science/hal-01184177}

Submitted on 17 Feb 2020

HAL is a multi-disciplinary open access archive for the deposit and dissemination of scientific research documents, whether they are published or not. The documents may come from teaching and research institutions in France or abroad, or from public or private research centers.
L'archive ouverte pluridisciplinaire HAL, est destinée au dépôt et à la diffusion de documents scientifiques de niveau recherche, publiés ou non, émanant des établissements d'enseignement et de recherche français ou étrangers, des laboratoires publics ou privés. 


\title{
Resource Allocation in Downlink Non-orthogonal Multiple Access (NOMA) for Future Radio Access
}

\author{
Marie-Rita Hojeij $^{(1,2)}$, Joumana Farah ${ }^{(3)}$, Charbel Abdel Nour ${ }^{(2)}$, Catherine Douillard ${ }^{(2)}$ \\ ${ }^{(1)}$ Department of Telecommunications, Faculty of Engineering, \\ Holy-Spirit University of Kaslik, P.O. Box 446, Jounieh, Lebanon \\ (2) Telecom Bretagne, Department of Electronics, Lab-STICC - UMR 6285 \\ Technopôle Brest Iroise, CS 83818 - 29238 Brest Cedex, France \\ (3) Department of Electricity and Electronics, Faculty of Engineering, \\ Lebanese University, Roumieh, Lebanon
}

\begin{abstract}
This paper investigates a new strategy for radio resource allocation applying a non-orthogonal multiple access (NOMA) scheme. It calls for the cohabitation of users in the power domain at the transmitter side and for successive interference canceller (SIC) at the receiver side. Taking into account multi-user scheduling, subband assignment and transmit power allocation, a hybrid NOMA scheme is introduced. Adaptive switching to orthogonal signaling (OS) is performed whenever the non-orthogonal cohabitation in the power domain does not improve the achieved data rate per subband. In addition, a new power allocation technique based on waterfilling is introduced to improve the total achieved system throughput. We show that the proposed strategy for resource allocation improves both the spectral efficiency and the cell-edge user throughput. It also proves to be robust in the case of communications in crowded areas.
\end{abstract}

Index terms - non-orthogonal multiple access, power domain multiplexing, waterfilling, resource allocation.

\section{INTRODUCTION}

With the proliferation of Internet usages, future communication networks will have to face by 2020 a mobile traffic volume 500 times larger than today's [1]. These challenges are pushing the limits of the actual generation of cellular technology, and pointing toward a need for a $5^{\text {th }}$ generation. In this sense, new designs of radio access technology (RAT), in terms of spectrum management and multiple access techniques, become essential to accommodate such requirements $[2,3]$.

The 3.9 and $4^{\text {th }}$ generation $(4 \mathrm{G})$ of mobile communication systems, Long-Term Evolution (LTE) and LTE-Advanced, adopted orthogonal multiple access (OMA) based on OFDM or single carrier Frequency Division Multiple Access (SC-FDMA). Nevertheless, nonorthogonal multiple access (NOMA) scheme appears as a promising multiple access candidate for future radio access. It increases spectral efficiency by allowing cohabitation of users in the power domain [4].

So far, the majority of existing literature dealing with NOMA proposed new strategies for maximizing system throughput without optimizing the total amount of used bandwidth and without guarantying a requested user service data rate. System level-performance is mostly evaluated with respect to OMA, i.e., when a subband is orthogonally divided, in bandwidth and in power, between collocated users $[5,6,7]$.

Many strategies for the optimization of resource allocation in a wireless multiuser OFDM system have been proposed and have shown promising results $[8,9]$. However, such strategies have not been considered yet in a NOMA-based system where additional design constraints should be taken into account. Therefore, this work aims to propose a new algorithm for the optimization of resource allocation based on non-orthogonal cohabitation in the power domain, on top of the OFDM layer.

The main target is the improvement of spectral efficiency and cell-edge user throughput, i.e. user fairness. The proposed algorithm for dynamic assignment of available subbands aims to achieve two goals: first, reduce the amount of used bandwidth; second, improve capacity while trying to satisfy requested service data rate per user.

In order to achieve these targets, we propose a hybrid solution for subband allocation that consists of a dynamic switching from NOMA to Orthogonal Signaling (OS) every time the non-orthogonal cohabitation does not achieve desired goals. To further boost the proposed system performance, a new power allocation scheme based on waterfilling is proposed and evaluated. Our results confirm that the combination of a NOMA and an OS shows better performance than using exclusively a NOMA scheme. Note that in [6] for comparison purposes, switching from NOMA to OMA is performed, such that the proposed resource allocation algorithm can be either entirely based on NOMA or on OMA. No criterion is proposed to enable this switching in a dynamic way or to vary the signaling scheme from one subband to another.

The remainder of the paper is organized as follows: Section II gives a general description of NOMA with SIC. Then Section III details the proposed iterative method for spectrum optimization. Simulation results are given in Section IV, and Section V concludes the paper.

\section{BASIC DESCRIPTION OF NOMA WITH SIC}

This section describes the general concept of NOMA including user multiplexing or pairing at the transmitter and signal separation at the user terminal.

We assume throughout this paper a downlink system with a single transmitter and a single receiver antenna. We consider $K$ users per cell, and a frequency-selective scheduler, where system bandwidth is divided into $S$ subbands. For the sake of simplicity, only two users are selected from the subset $K$ to be scheduled over subband $s$ $(1 \leq s \leq S)$. The base station transmits a signal for user $i$ $(i=1,2)$, over subband $s, x_{s, i}$, with transmit power $P_{s, i}$. The 
transmit signal, $x_{s}$, over a subband $s$, can be written as:

$$
x_{s}=\sqrt{P_{s, 1}} x_{s, 1}+\sqrt{P_{s, 2}} x_{s, 2}
$$

The received signal of user $i$ over subband $s, y_{s, i}$, is represented by:

$$
y_{s, i}=h_{s, i} x_{s}+w_{s, i}
$$

where $h_{s, i}$ is the frequency domain complex channel coefficient between user $i$ and the base station over subband $s . w_{s, i}$ represents the Gaussian noise in addition to the inter-cell interference of user $i$ over subband $s$.

At the receiver side, multi-user signal separation is conducted using a SIC process. The optimal order for SIC decoding is in the increasing order of user channel gains. We assume that a user can correctly decode signals of users with earlier decoding order. In other words, user $j$ can successfully remove the inter-user interference of user $i$ whose $h_{i}^{2}$ is lower than $h_{j}^{2}[10,11]$. In our case, when two users are multiplexed over subband $s$, and assuming that $h_{s, 2}^{2}$ is lower than $h_{s, 1}^{2}$, user 2 does not require SIC since it comes first in the decoding order, it treats signal $x_{s, 1}$ intended for user 1 as noise. As for user 1, it first decodes signal $x_{s, 2}$ intended for user 2 and subtracts its component from the received signal $x_{s}$. Then it decodes its own signal without interference from $x_{s, 2}$. Assuming successful decoding and no error propagation, the throughput of user $i(i=1,2)$ over subband $s, R_{s, i}$, is given by:

$$
\begin{gathered}
R_{s, 1}=\frac{B}{S} \log _{2}\left(1+\frac{P_{s, 1} h_{s, 1}^{2}}{N_{0} \frac{B}{S}}\right), \\
R_{s, 2}=\frac{B}{S} \log _{2}\left(1+\frac{P_{s, 2} h_{s, 2}^{2}}{P_{S, 1} h_{s, 2}^{2}+N_{0} \frac{B}{S}}\right),
\end{gathered}
$$

where $B$ represents the total system bandwidth, $S$ denotes the maximum number of available subbands, and $N_{0}$ is the power spectral density of the additive white Gaussian noise (assumed to be constant over all subbands).

It can be seen from (3) and (4) that the choice of the multiplexed users over subband $s$ and the amount of allocated power for each user significantly affect user throughput performance. For this aim, multi-user scheduling and multi-user power allocation techniques are proposed and evaluated.

\section{DESCRIPTION OF THE PROPOSED ITERATIVE METHOD FOR RESOURCE ALLOCATION}

Resource allocation for a non-orthogonal system should consider the following additional design constraints: The choice of user pairing, the power distribution between allocated subbands and the power division between paired users within a subband. The allocation technique in fig. 1 tries to answer favorably all of these design constraints.

\section{A. Formulation of the resource allocation problem}

In addition to maximizing system throughput as performed in the majority of existing literature on NOMA, this work targets minimizing the amount of used bandwidth. In other words, the proposed allocation technique tends to provide to each user its requested data rate with the minimum number of subbands, under the constraint of the maximum allowed transmit power.

Let $K$ be the number of users that need to communicate, $S_{A}$ the actual number of available subbands $\left(1 \leq S_{A} \leq S\right)$, i.e. $S-S_{A}$ subbands are supposed to be occupied by another system, $R_{k \text {,requested }}(1 \leq k \leq K)$ the download data rate requested by user $k$ from the base station, $P_{s, k}$ the transmit power over subband $s$ allocated to user $k\left(P_{s, k} \neq 0\right.$ if $k$ is scheduled on s), $R_{s, k}$ the achieved data rate by user $k$ over subband $s$, and $S_{k}$ the set of all subbands allocated to user $k$. The optimization problem can be formulated as follows:

$\underset{P_{s, k}, S_{k}}{\operatorname{minimize}} \sum_{k=1}^{K} \operatorname{card}\left(S_{k}\right)$

\section{Subject to}

$\sum_{s \in S_{k}} R_{s, k}=R_{k, \text { requested }} \quad \forall k, 1 \leq k \leq K$

$\sum_{k}\left(\sum_{s \in S_{k}} P_{s, k}\right) \leq P_{\max }$

$P_{s, k} \geq 0, \forall s \in S_{k}, 1 \leq k \leq K$

where $\operatorname{card}\left(S_{k}\right)$ represents the cardinality of the set of subbands allocated to user $k$.

If user $k$ has a channel gain over $s$ that allows him to perform SIC, his data rate is computed based on Eq. (3). Otherwise, it is computed based on Eq. (4).

Eq. (5) represents the main design function. It tries to minimize the number of allocated subbands under the constraints shown in Eq. (6), (7), and (8)

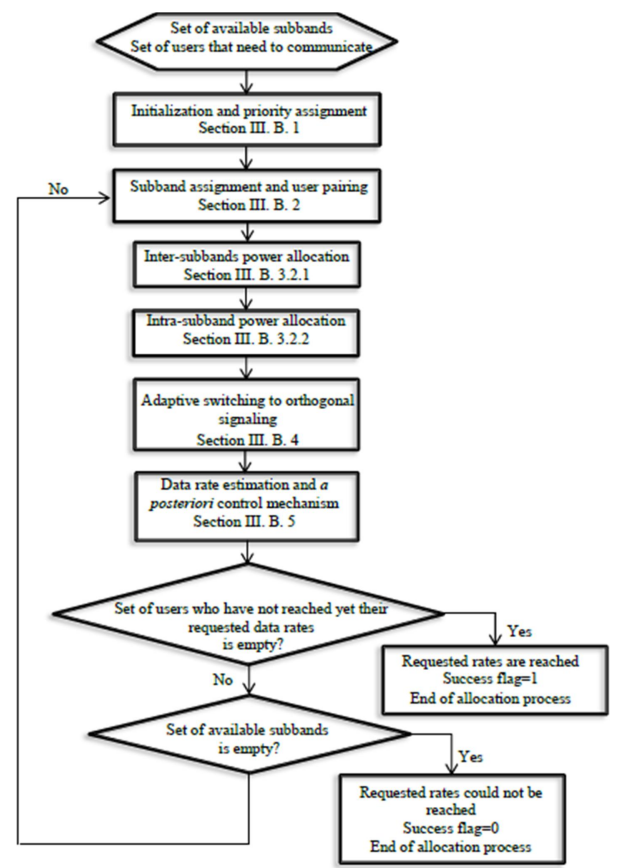

Fig. 1. Proposed allocation algorithm

\section{B. The proposed algorithm for resource allocation 1. Initialization and priority assignment}

In order to search for a global solution, it is necessary to have the full channel gain information available, i.e. channel gain between cellular user and BS. $\mathbf{H}$ is an $S_{A} \times K$ matrix provided in fig. 2, where $h_{s, k}$ is the channel gain experienced on subband $s$ by user $k$. Since at the beginning of the allocation process, transmit powers $P_{s, k}$, and user 
rates $R_{s, k}$ are all set to zero, priorities are defined based on the channel gain matrix $\mathbf{H}$ :

- For each user $k$, select the highest channel gain $h_{s_{b e s t}, k}$ among the elements in the $k^{\text {th }}$ column of matrix $\mathbf{H}$ (denoted by a circle in fig. 2)

- The user with highest priority (lowest priority) is the one having the lowest (highest) channel gain among circled elements.

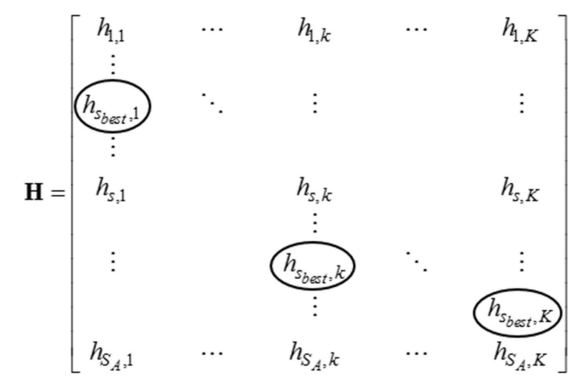

Fig. 2. Channel gain matrix $\mathbf{H}$

\section{Subband assignment and user pairing}

During iteration, users to be paired together over an assigned subband $s_{f}$ are identified by applying the following steps:

\section{Step 1: User selection}

Select user $k_{1}$ among the set of users that need to communicate based on:

\section{Choice criterion 1:}

While it exists at least 2 users whose data rates are zero, select among them user $k_{1}$ based on the priority constraints defined in section III.B.1.

\section{Choice criterion 2:}

Once data rates of all users are non-zero, select user $k_{1}$ showing the largest rate distance or gap towards its requested service data rate.

\section{Step 2: Subband assignment}

Attribute the most favorable subband denoted by $s_{f}$, to user $k_{1}$. Then, $s_{f}$ is removed from the set of available subbands.

\section{Step 3: User pairing}

Select user $k_{2}$ to be multiplexed in the power domain with user $k_{1}$ on the current subband $s_{f}$. User pairing can be done in several ways. We have evaluated two options:

\section{Pairing 1:}

User $k_{2}$ is chosen as the user having the next lowest channel gain over $s_{f}$ when compared to the one of $k_{1}$.

\section{Pairing 2:}

User $k_{2}$ is chosen as the user having the worst channel gain over $s_{f}$.

In the two pairing options, the channel gain of user $k_{2}$ is chosen to be less than that of $k_{1}$. Therefore, user $k_{2}$ does not perform SIC. Instead, his corresponding receiver considers the signal of user $k_{1}$ as interfering noise with $P_{s, k_{1}} h_{s, k_{2}}^{2}$ as the interfering term.

\section{Step 4: Inverting roles}

If during the allocation process, it happens that user $k_{1}$ has the lowest gain on its attributed subband $s_{f}$, user $k_{2}$ is then chosen as the user having the highest gain on this subband, if pairing 2 is adopted in step 3. Otherwise, it will be chosen as the user having the next highest channel gain over $s_{f}$ when compared to the one of $k_{1}$.

\section{Multi-user power allocation}

\subsection{Optimum power allocation}

In $[5,10]$, static allocation is used where the total transmit power is identically divided between subbands. However, it is stated that the resulting achievable throughput is penalized since waterfilling is not used. Therefore, we propose to apply a waterfilling-based subband power allocation. It takes into consideration the channel gains of the two paired users within each subband. It is described by the following optimization problem:

At each stage of the allocation process, maximize the total achieved throughput for users that have not yet reached their requested data rate under the constraint of the total remaining power:

$\underset{\left\{P_{s, k_{1}}, P_{s, k_{2}}\right\}}{\operatorname{maximize}} \sum_{s \in S_{u}}\left(R_{s, k_{1}}+R_{s, k_{2}}\right)$

Subject to

$\sum_{s \in S_{u}} P_{s}=P_{r e m}$

$S_{u}$ is the set of subbands attributed to users whose requested data rates have not been reached so far (those users constitute a set $U$ ), and $P_{\text {rem }}$ denotes the remaining transmit power to be distributed between subbands, at a certain stage of the allocation algorithm.

Solving this optimization problem using Lagrange multipliers leads to the following formulation of the objective function, where $\lambda$ is the Lagrange multiplier:

$$
\begin{aligned}
J= & \sum_{s \in S_{u}} \frac{B}{S} \log _{2}\left(1+\frac{P_{s, k_{1}} h_{s, k_{1}}^{2}}{N_{0} \frac{B}{S}}\right)+\sum_{s \in S_{u}} \frac{B}{S} \log _{2}\left(1+\frac{P_{s, k_{2}} h_{s, k_{2}}^{2}}{P_{s, k_{1}} h_{s, k_{2}}^{2}+N_{0} \frac{B}{S}}\right) \\
& +\lambda\left(P_{r e m}-\sum_{s \in S_{u}}\left(P_{s, k_{1}}+P_{s, k_{2}}\right)\right)
\end{aligned}
$$

In $[5,12,13]$ related to NOMA, power multiplexing is done such that the highest power is given to the user with the weakest channel (user $k_{2}$ in our case). Therefore, we adjust the power allocation ratio between $P_{s, k_{1}}$ and $P_{s, k_{2}}$ by setting a dynamic parameter $\beta_{s}$ such that:

$$
P_{s, k_{2}}=\frac{1-\beta_{S}}{\beta_{S}} P_{s, k_{1}} \text {, with } \frac{1}{2}<\beta_{S}<1
$$

By substituting (12) in (11), then differentiating $J$ with respect to $P_{s, k_{1}}$ and $\lambda$, and by setting the result to zero, we obtain a non-linear system of $N_{u}+1$ equations with $N_{u}+1$ unknowns $P_{s, k 1}$ and $\lambda$, where $N_{u}$ is the current number of elements in $S_{u}$.

\subsection{Sub-optimum waterfilling-based power allocation}

The optimum solution performs a waterfilling-based allocation while considering channel gains of all paired users. This reveals to be impractical and complex to consider. Therefore, we propose a sub-optimum solution, where the power is allocated among users in two stages:

\subsubsection{Stage 1: Inter-subband power allocation}

We propose to consider only the highest channel state within each subband. In other words, the highest channel gain on a subband determines the total amount of power that will be attributed to it, using a waterfilling process, and that will be subsequently partitioned between the two 
paired users. The waterfilling process is performed in an iterative way as in [8]. Even though this allocation technique represents a sub-optimum solution, it is expected to perform better than static power allocation.

\subsubsection{Stage 2: Intra-subband power allocation}

Power is now to be partitioned between paired users within each subband. Intra-subband repartition could be dynamic, based on paired users channel gains, or static, according to a static threshold.

\section{Static intra-subband power allocation: Fixed Power} Allocation (FPA)

The repartition is done in a static way over all subbands, where the total transmit power on subband $s, P_{s}$, is divided between paired users according to $\left(\beta . P_{s},(1-\beta) P_{s}\right)$, with $\beta$ $(0 \leq \beta \leq 0.5)$ being a constant parameter over all subbands, and $P_{s}$ being the total transmit power allocated in stage 1 to subband $s$. The user with the highest channel gain will be given $\beta . P_{s}$ and the paired user will be given the rest.

Dynamic intra-subband power allocation: Fractional transmit power allocation (FTPA)

The repartition is done in a dynamic way, similar to the fractional transmit power allocation algorithm (FTPA) in [5] which is based on the channel gains of the two multiplexed users, such that $\beta_{s}$ in (12) is given by:

$$
\beta_{s}=\frac{h_{s, k_{1}}^{-2 \alpha}}{h_{s, k_{1}}^{-2 \alpha}+h_{s, k_{2}}^{-2 \alpha}},
$$

where $\alpha(0 \leq \alpha \leq 1)$ is a decay factor that accounts for the amount of power attributed to user $k_{2}$ (this amount is increased with $\alpha$ ). $\alpha$ is kept constant over the subbands and is determined a priori via computer simulations, such that the achieved spectral efficiency is maximized.

\section{Adaptive switching to orthogonal signaling}

Improvement in spectral efficiency thanks to NOMA is not systematic. Indeed, sometimes the loss in data rate experienced by user $k_{1}$, when sharing its subband with user $k_{2}$ is greater than the data rate gain achieved by $k_{2}$. In this case, NOMA is not the appropriate solution; we propose to allocate this subband to user $k_{1}$ alone.

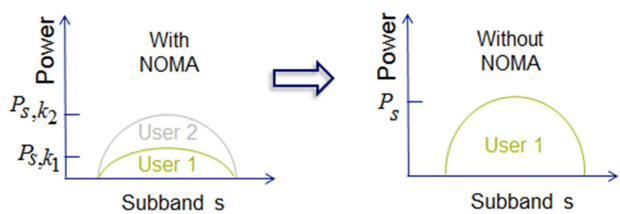

Fig. 3. Adaptive switching from NOMA to OS

The decision to switch to OS can be made by testing the following condition:

$$
\gamma\left(R_{S}-R_{S, k_{1}}\right)>R_{S, k_{2}}
$$

with:

$$
R_{S}=\frac{B}{S} \log _{2}\left(1+\frac{P_{S} h_{S, k_{1}}^{2}}{N_{0} \frac{B}{S}}\right) \text {, without NOMA. }
$$

When condition (14) is satisfied, the allocation automatically switches to orthogonal signaling, for the current subband s. $\gamma(0<\gamma<1)$ is a parameter to be determined a priori via simulations such that the achieved spectral efficiency is maximized. Results in IV provide insight into the sensitivity of system performance to the values of $\gamma$. In general, with increasing values of $\gamma$ the allocation process tends to switch to orthogonal signaling.

\section{Data rate estimation and control mechanism}

At the end of a subband assignment with its power allocation to users $k_{1}$ and $k_{2}$, the algorithm updates corresponding data rates. Then, it verifies if user $k_{1}$ reaches its requested data rate, that is if the actual total data rate of user $k_{1}, R_{k_{1}, \text { tot }}$, is equal to $R_{k_{1}, \text { requested }}$. When true, user $k_{1}$ is removed from the set $U$. Then, the allocated power values on subbands assigned to user $k_{1}$ (for $k_{1}$ and the paired users) are kept unvaried for the rest of the allocation process. The remaining power $P_{\text {rem }}$ is updated by subtracting the power allocated to the subbands that have just been assigned and removed from the set $S_{u}$.

When the actual data rate is higher than the requested data rate $\left(R_{k_{1}, \text { tot }}>R_{k_{1}, \text { requested }}\right)$, the total amount of power allocated to user $k_{1}$ should be reduced in such a way to reach the requested data rate. Among the subbands allocated to user $k_{1}$ that remain modifiable (not paired with a user that reached its requested rate), we adjust the power on subband $s_{a}$ having the least channel amplitude. A similar procedure is applied on user $k_{2}$ if it reaches its requested data rate. When adjusting the transmit power of user $k_{1}$ on subband $s_{a}$, we encounter two cases:

The first case occurs when user $k_{1}$ exhibits the highest channel gain over $s_{a}$. The adjustment follows:

First, the transmission rate of $k_{1}$ over $s_{a}$ is estimated using:

$$
R_{S_{a}, k_{1}}=\frac{B}{S} \log _{2}\left(1+\frac{P_{s_{a}, k_{1}} h_{s_{a}, k_{1}}^{2}}{N_{0} \frac{B}{S}}\right)
$$

Then, this rate is subtracted from the actual total rate of user $k_{1}$, yielding:

$$
R_{r e m}=R_{k_{1}, t o t}-R_{S_{a}, k_{1}}
$$

Now, the necessary data rate on $s_{a}$ is estimated as: $R_{k_{1}, \text { requested }}-R_{\text {rem }}$. The power of user $k_{1}$ over $s_{a}$ is modified in such a way to yield the above estimated data rate.

$$
P_{s_{a}, k_{1}}=\frac{2^{\left(R_{k_{1}}, \text { requested }-R_{\text {rem }}\right) \frac{S}{B}-1}}{h_{s_{a}, k_{1}}^{2}} N_{0} \frac{B}{S}
$$

The power of the collocating user should be reduced (eq. (12)) in order to maintain the same power ratio $\left(1-\beta_{S_{a}}\right) / \beta_{S_{a}}$

For the second case, when user $k_{1}$ exhibits the lowest channel gain over $s_{a}$, power adjustment is done by modifying eq. (15) using eq. (4), eq. (16) is kept the same, and eq. (17) is replaced by (18) using eq. (12) and (4):

$$
P_{s_{a}, k_{1}}=\frac{\left(2^{\left(R_{k_{1}}, \text { requested }-R_{\text {rem }}\right) \frac{S}{B}}-1\right) N_{0} \frac{B}{S}}{\left(1-\left(\left(2^{\left(R_{k_{1}}, \text { requested }-R_{\text {rem }}\right) \frac{S}{B}}-1\right) \frac{\beta_{s_{a}}}{1-\beta_{S_{a}}}\right)\right) h_{s_{a}, k_{2}}^{2}}
$$

Sometimes, when trying to adjust the power of user $k_{l}$ over $s_{a}$, it can happen that $R_{\text {rem }}$ is still greater than $R_{k_{1}, \text { requested }}$ In this case, another subband, having a channel gain higher than that of $s_{a}$, is chosen for power adjustment. 


\section{NUMERICAL RESULTS}

\section{A. Performance evaluation}

In this paper, we consider three important system-level performance indicators: the achieved system capacity, the amount of used bandwidth, and the cell-edge user throughput. The first two indicators can be merged into a single metric: spectral efficiency, calculated as:

Spectral_Efficiency $=\frac{\text { Achieved system capacity }}{\text { Amount of used bandwidth }}$

In order to assess the performance gain of our proposed hybrid solution NO_O_WF based on a combination of NOMA and OS with waterfilling as power allocation scheme, we compare it to three reference scenarios:

- NO_WF: In this scenario, switching to OS is not allowed. The allocation process is purely based on NOMA and waterfilling is used for power allocation.

- O_WF: Only OS is applied and non-orthogonal cohabitation is not allowed. Waterfilling is used as power allocation scheme.

- NO O EP: The combination of NOMA and OS is applied with a static-based power allocation scheme where power is equally divided among subbands.

\section{B. Simulation results}

Simulations were first performed to validate the choices of different design parameters in terms of user pairing, multi-user power allocation and adaptive switching. Then, robustness of the proposed system in the case of communication in crowded areas is evaluated.

In our simulation setup, $K$ users are randomly positioned following a uniform distribution in a $10 \mathrm{~km}$ radius cell with a maximum path loss difference of $20 \mathrm{~dB}$ between users. $K$ varies between 5 and 20 . System bandwidth $B$ is $100 \mathrm{MHz}$, the maximum number of available subbands is 128 , the total transmit power of the Base Station is 1000 $\mathrm{mW}$, and the user requested data rate is set to $5 \mathrm{Mbps}$. The noise power spectral density is $4 \cdot 10^{-18} \mathrm{~W} / \mathrm{Hz}$. The transmission medium is modeled by a frequency-selective Rayleigh fading channel with a root mean square delay spread of $500 \mathrm{~ns}$. Perfect knowledge of the channel gains of all users by the BS is assumed in this study.

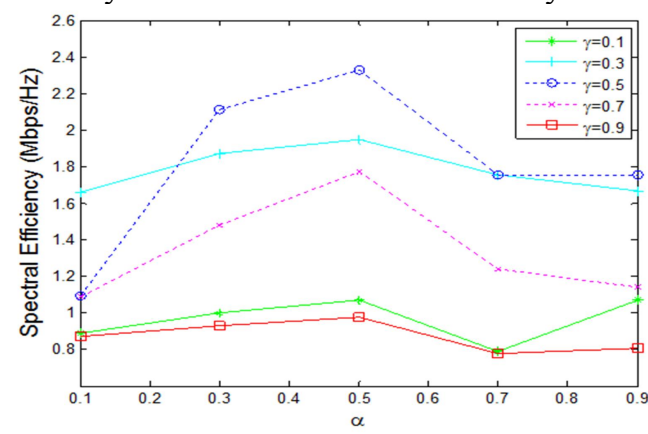

Fig. 4. Spectral efficiency of NO_O_WF for values of $\alpha$ and $\gamma$.

First, we start by identifying the values of the FTPA decay factor represented by $\alpha$ and of the adaptive switching to OS parameter denoted by $\gamma$. Fig. 4 shows the obtained spectral efficiency when NO_O_WF is evaluated for different $\alpha$ and $\gamma$ values with $\bar{K}=1 \overline{0}$ and the actual number of available subbands $S_{A}$ is equal to $128\left(S_{A}=S\right)$. Spectral efficiency is maximized for $\alpha=0.5$ and $\gamma=0.5$.
Similar optimal values were observed for different values of $K$ and $S_{A}$. Therefore, these values of $\alpha$ and $\gamma$ are adopted in the remainder of the study.

Then, the impact of user pairing and intra-subband power allocation strategies on system performance is evaluated, for $S_{A}=128$. Fig. 5 shows the spectral efficiency of NO_O_WF when FTPA and FPA are used. The effect of the two pairing techniques presented in section III.B. 1 is also shown on the same graph. We notice that the combination between FTPA and pairing 2 outperforms FPA (for different values of $\beta$ ), with a gain ranging from $16 \%$ when the number of users is high, up to $40 \%$ when the number of users per cell is equal to 5 .

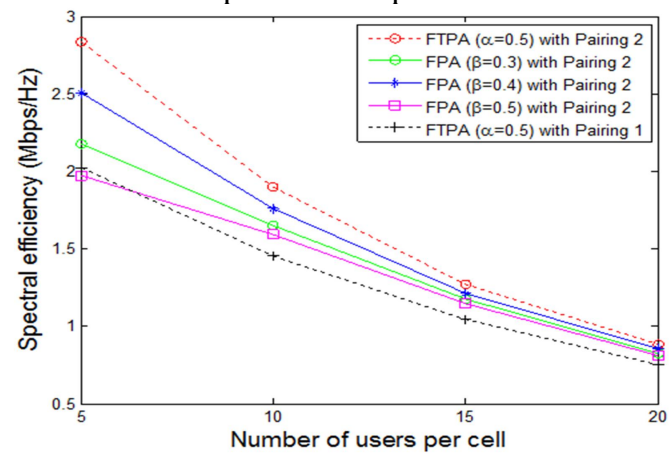

Fig. 5. Spectral efficiency of NO_O_WF for dynamic and fixed intra-subband power allocation schemes, and for different channel gain difference between paired users.

Since users having the largest possible gain difference within each subband are paired together (pairing 2), the power-difference between their received signals should be large too thanks to the application of FTPA. Therefore, the inter-user interference experienced by user $k_{2}\left(P_{s, k_{1}} h_{s, k_{2}}^{2}\right)$ is reduced, not only due to the choice of user $k_{2}\left(h_{s, k_{2}}^{2}\right)$ but also since the power of user $k_{1}$ 's signal, $P_{s, k_{1}}$, is lowered.

The performance of our technique is investigated in the context of a congested area for two different setups:

Case 1: The number of users per cell is equal to 10 and the actual number of available subbands ranges from 16 to 128 , with a fixed subband bandwidth at $100 / 128 \mathrm{MHz}$.

Case 2: The actual number of available subbands is 128 $\left(S_{A}=S\right)$ and the number of users per cell is varied between 5 and 20 .

Fig. 6 compares the spectral efficiency of the simulated scenarios, according to case 1 (left) and to case 2 (right).

In both cases, our technique outperforms the other simulated methods. The gain in spectral efficiency is due to several factors:

- The reduction in the amount of used bandwidth due to non-orthogonal cohabitation in the power domain makes NO_WF outperform O_WF.

- The improvement in system capacity due to waterfilling process helps NO_WF outperform NO_EP

- The use of a dynamic adaptive switching to orthogonal-based system improves NO_O_WF performance with respect to NO_WF.

For 32 available subbands, and for 10 users per cell, $\mathrm{NO} \mathrm{O} W \mathrm{~W}$ has a spectral efficiency of $2.2 \mathrm{Mbps} / \mathrm{Hz}$ compared to $2.02,1.85$, and $1.5 \mathrm{Mbps} / \mathrm{Hz}$ with $\mathrm{NO}_{-} \mathrm{WF}$, 
NO_EP and O_WF, respectively. When the number of available subbands decays, performance and reduced bandwidth advantages of NO_O_WF are kept. For example, when this number drops to 16 , the measured spectral efficiency remains in favor of the proposed technique and is respectively $2.9,2.8,2.7$, and 2.4 $\mathrm{Mbps} / \mathrm{Hz}$ for NO_O_WF, NO_WF, NO_EP, and O_WF.
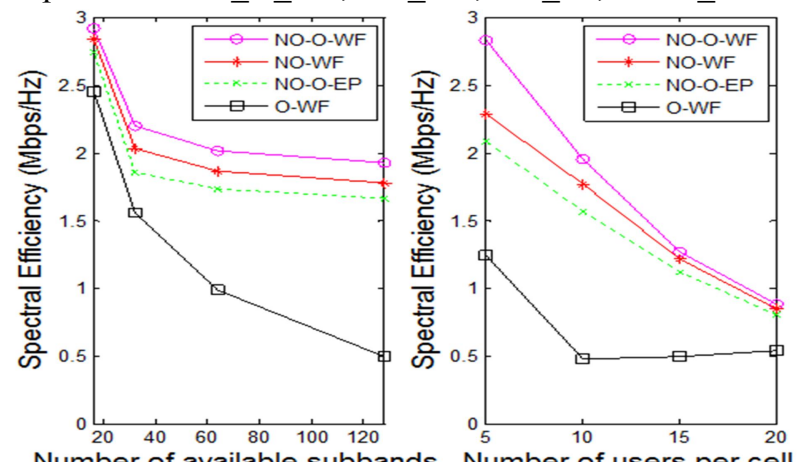

Number of available subbands Number of users per cell

Fig. 6. Spectral efficiency comparison as a function of the number of available subbands (left), and as a function of the number of users per cell (right).

The cell-edge user throughput is an important fairness evaluator of an allocation process. Fig. 7 shows this metric as a function of the number of users per cell, where the number of available subbands is fixed to 128 .

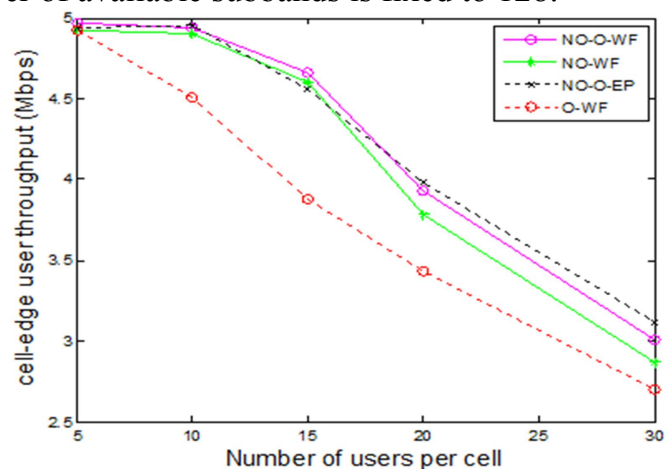

Fig. 7. Cell-edge user throughput of INO_WF, IO_WF, and INO EP as a function of the number of users per cell

The cell-edge user throughput in the case of NOMA is almost $20 \%$ higher than in the case of orthogonal signaling. In addition, the proposed hybrid solution NO_O_WF always outperforms NO_WF. Indeed, taking into account channel state information while assigning priorities in section III.B.1 introduces high fairness to the allocation process. The proposed algorithm improves user fairness while outperforming orthogonal signaling in terms of spectral efficiency.

When the number of users per cell is limited, waterfilling-based power allocation shows a higher celledge user throughput compared to equal power allocation. However, when the number of users per cell becomes large, the success rate (i.e. the probability of succeeding to respect required data rates by all users) decreases. Therefore, uniform power allocation reveals to be the best choice. This is due to the fact that waterfilling-based algorithms generally optimize the average throughput and may not give the best fairness to the cell-edge user, especially for large values of $K$. Nevertheless, our approach still presents an important gain in performance compared to orthogonal signaling in terms of spectral efficiency and degree of fairness.

\section{CONCLUSION}

This paper introduces a new strategy for subband and power allocation under a non-orthogonal multiple access scenario. It targets minimizing spectrum usage while satisfying requested user data rates. The choice of user pairing, waterfilling-based inter-subband power allocation, adaptive intra-subband power allocation and dynamic switching from NOMA to orthogonal signaling represent design parameters. Simulation results show that the proposed method allows a significant increase in spectral efficiency, compared to a system purely based on orthogonal or non-orthogonal signaling. We are currently undergoing further research to incorporate an optimal solution for power allocation within our iterative technique and to study its applicability to the context of an uplink transmission.

\section{REFERENCES}

[1] NTT DOCOMO, "Requirements, candidate solutions \& technology roadmap for LTE Rel-12 onward," 3GPP RWS120010, June 2012.

[2] Y. Kishiyama, A. Benjebbour, H. Ishii, and T. Nakamura, "Evolution concept and candidate technologies for future steps of LTE-A". IEEE Inter. Conf. on Comm. Syst., Nov 2012.

[3] G. Wunder et al, "5GNOW: Non-Orthogonal, Asynchronous Waveforms for Future Mobile Applications", IEEE Comm. Magazine, February 2014, pp. 97-105.

[4] Sharp corporation, "Evolving RAN Towards Rel-12 and beyond," RWS-120039, 3GPP Workshop on Release 12 Onward Ljubljana, Slovenia, June 11 - 12, 2012.

[5] A. Benjebbour, A. Li, Y. Saito, Y. Kishiyama, A. Harada, and T. Nakamura, "System-level performance of downlink NOMA for future LTE enhancements," IEEE Globecom, Dec. 2013.

[6] A. Benjebbour, Y. Saito, Y. Kishiyama, A. Li, A. Harada, and T. Nakamura, "Concept and practical considerations of nonorthogonal multiple access (NOMA) for future radio access," ISPACS 2013, Nov. 2013.

[7] Y. Saito, Y. Kishiyama, A. Benjebbour, T. Nakamura, A. Li, and K. Higuchi, "Non-orthogonal multiple access (NOMA) for future radio access," IEEE VTC spring 2013, June 2013.

[8] J. Farah and F. Marx, "Combining strategies for the optimization of resource allocation in a wireless multiuser OFDM system," AEU Inter. Journal of Elect. and Comm., vol. 61, no. 10, pp. $665-677,2007$.

[9] S. Han, H. Kim, Y. Han, J. M. Cioffi and V. C. M. Leung, "A distributed power allocation scheme for sum-rate maximization on cognitive GMACs," in Proc. of PIMRC, pp. 1854-1858, Sept. 2010.

[10] S. Tomida and K. Higuchi, "Non-orthogonal Access with SIC in Cellular Downlink for User Fairness Enhancement," Inter. Symp. on Intell. Signal Process. and Comm. Systems (ISPACS), pp.1-6, Dec. 7-9 2011.

[11] T. Takeda and K. Higuchi, "Enhanced user fairness using non orthogonal access with SIC in cellular uplink," IEEE VTC Fall 2011, Sept. 2011.

[12] B. Kim, S. Lim, H. Kim, S. Suh, J. Kwun, S. Choi, C. Lee, S. Lee, and D. Hong," Non-orthogonal Multiple Access in a Downlink Multiuser Beamforming System”, MILCOM 2013.

[13] D. Tse and P. Viswanath, Fundamentals of Wireless Communication, Cambridge University Press, 2005. 